\title{
Assessing the Clinical Impact of Palmar-Plantar Erythrodysesthesia in Patients Receiving Capecitabine Monotherapy
}

\section{Wood $\mathrm{J}^{1 *}$ and Anne Thomas ${ }^{2}$}

${ }^{1}$ Academic Clinical Lecturer in Medical Oncology, Department of Cancer Studies, Robert Kilpatrick Clinical Sciences Building, University of Leicester, Leicester Royal Infirmary, Leicester, UK

${ }^{2}$ Professor of Cancer Therapeutics, Department of Cancer Studies, University of Leicester, Level 2, Osborne Building, Leicester Royal Infirmary, Leicester, UK

\begin{abstract}
Capecitabine is an oral fluoropyrimidine with antineoplastic activity indicated for treating colorectal and breast cancer either as monotherapy or in combination with other drugs. Palmar-plantar Erythrodysesthesia (PPE) is one of the most commonly reported side-effects of this treatment however its actual incidence and the subsequent dosing changes that it may lead to in the clinic is poorly reported. To gain more information about the clinical impact of PPE we conducted an audit of all patients treated with capecitabine monotherapy at our centre during 2014. Ninety patients were identified as receiving at least one dose of capecitabine treatment. PPE was the documented reason for dose reduction in $28(31 \%)$ of these individuals. In addition, $7(20 \%)$ of the breast cancer patients were converted from a 3 weekly to a less intense 4 weekly alternate dose regimen. In the colorectal cancer patients, PPE was more likely to lead to a dose reduction in those being treated with curative rather than palliative intent ( $49 \%$ versus $29 \%$ ). An effective treatment for PPE has the potential to improve quality of life and outcomes in patients being treated with capecitabine chemotherapy.
\end{abstract}

Keywords: Capecitabine; Chemotherapy; Colorectal cancer; Breast cancer; Hand-foot syndrome; Palmar-plantar erythrodysesthesia

\section{Aim}

To establish the incidence of PPE and its effects on dose intensity in patients being treated with capecitabine monotherapy.

\section{Introduction}

Capecitabine is an oral fluoropyrimidine with antineoplastic activity indicated for treating colorectal and breast cancer either as monotherapy or in combination with other drugs (XELODA Prescribing Information). It is absorbed intact in the intestine and converted in the body to 5 -fluorouracil (5-FU) by a three step enzymatic cascade. Its mechanism of activation is unique in that it exploits the high activity of Thymidine Phosphorylase (TP) in malignant cells, resulting in the generation of 5-FU preferentially in tumour tissue itself [1].

The recommended dose of capecitabine monotherapy is $1250 \mathrm{mg} /$ $\mathrm{m}^{2}$ administered orally twice daily (morning and evening; equivalent to $2500 \mathrm{mg} / \mathrm{m}^{2}$ total daily dose) for 2 weeks followed by a 1-week rest period given as 3 -week cycles. In the adjuvant treatment (i.e., given after surgery with curative intent) of Colorectal Cancer (CRC), 8 cycles (24 weeks) of treatment are recommended [2]. In the palliative treatment (i.e., for incurable disease when the intent is to improve symptoms and prolong life) of breast cancer and CRC, the treatment duration may be extended if patients are both tolerating treatment well and clinically benefiting. Capecitabine may also be used concurrently with radiotherapy at the lower dose of $825 \mathrm{mg} / \mathrm{m}^{2}$ for the neoadjuvant treatment of rectal cancer [3].

As with all chemotherapies, its use is limited by toxicities. The most common adverse reactions reported in clinical trials $(\geq 30 \%)$ are, Palmar-Plantar Erythrodysesthesia (PPE), diarrhoea, nausea, vomiting, abdominal pain, fatigue/weakness, and hyperbilirubinemia $[2,4,5]$.

PPE, also known as hand foot syndrome, Burgdorf's reaction or chemotherapy-induced acral erythema is a dermatological reaction that appears on the palms of the hand and/or the soles of the feet. It is a recognised side-effect of several anticancer agents including: capecitabine, docetaxol, cytarabine, sunitinib and sorafenib [6,7]. The exact pathogenic mechanism has not yet been fully identified and is probably different for the diverse range of agents associated with the condition. Doxorubicin and sorafenib have been postulated to cause PPE due to local delivery of high drug concentrations though eccrine glands which have their highest density in the palms and soles [8,9]. PPE may also develop preferentially due to the increased vascularization, temperature and pressure in the hands and feet in comparison to other areas of the body [10]. It has also been proposed that keratinocytes in the skin of the palms and soles may contain increased levels of TP, which leads to the production and accumulation of 5-FU through local capecitabine metabolic activation [11].

PPE has been reported to be the most common adverse effect of capecitabine containing chemotherapy affecting up to $82 \%$ of patients at some point during their course of treatment [12]. It is more likely to develop and occur with greater severity as the number of chemotherapy cycles and thus duration of treatment increases.

PPE typically presents with erythematous plaques of the hands and soles of the feet. In the most commonly used criteria - the National Cancer Institute's Common Terminology Criteria for Adverse Events (NCI CTCAE version 4) [13] it is classified as grades 1-3 with increasing severity as follows:

*Corresponding author: Wood J, Academic Clinical Lecturer in Medical Oncology, Department of Cancer Studies, Robert Kilpatrick Clinical Sciences Building, University of Leicester, Leicester Royal Infirmary, Leicester, UK, Tel: 4401162525480; E-mail: jpw8@le.ac.uk

Received February 08, 2016; Accepted March 05, 2016; Published March 15 2016

Citation: Wood J, Thomas A (2016) Assessing the Clinical Impact of PalmarPlantar Erythrodysesthesia in Patients Receiving Capecitabine Monotherapy. Med Rep Case Stud 1: 107. doi: 10.4172/2572-5130.1000107

Copyright: ( 2016 Wood J, et al. This is an open-access article distributed under the terms of the Creative Commons Attribution License, which permits unrestricted use, distribution, and reproduction in any medium, provided the original author and source are credited. 
- Grade 1-numbness, dysesthesia/paresthesia, tingling, painless swelling or erythema not disrupting normal activities.

- Grade 2-painful erythema with swelling that affects daily activities.

- Grade 3-moist desquamation, ulceration, blistering or severe pain leading to an inability to work or perform daily activities.

As the pathogenesis of PPE has not been clarified, no effective treatment has been discovered to date [12]. Currently, most patients are advised about non-pharmacological measures including the avoidance of pressure and prevention of injury. Simple emollients to hydrate the skin are also often prescribed but in the event of recurrent episodes or an increase in its severity $\geq$ grade 2; dose reductions, delays or even stoppage of chemotherapy may be needed. This has the potential to reduce the effectiveness of the treatment. Only a few randomised trials have investigated pharmacological preventive strategies for capecitabine associated PPE. Empiric treatment with pyridoxine has been shown to be ineffective $[12,14]$ and similarly uric acid based creams have been ineffective when compared to a placebo [15]. Uric acid based cream has however been recommended as a reasonable standard for future studies having shown superiority to an antioxidant cream but this study can be criticised for lacking a placebo arm [16]. Celecoxib may reduce the incidence of PPE [17,18] but this drug bears the risk of cardiac toxicity and the positive results have not been confirmed in other studies [16].

In clinical trials, PPE has been demonstrated to cause treatment interruption or dose reduction of the capecitabine in between $13 \%$ to $31 \%$ of patients $[5,19]$. In metastatic breast cancer, when patients are potentially taking this treatment for many months and quality of life is of paramount importance, alternative schedules e.g. 7 days on followed by 7 days off treatment have been investigated and shown to be tolerable although the efficacy of this altered schedule remains unknown [20,21].

Although it is well documented in clinical trials that capecitabine causes PPE, its actual incidence and the subsequent dosing changes that this may lead to in the clinic is poorly reported. To gain more information about the incidence and clinical impact of PPE we conducted an audit of patients treated with capecitabine monotherapy at our centre.

\section{Method}

A retrospective audit was performed on all patients who had received capecitabine monotherapy at Leicester Royal Infirmary at any time during 2014 for any indication. It was anticipated that by including all patients who received this drug the data would be representative of the entire population of patients suitable to receive this treatment. Patients receiving capecitabine at the lower dose of $825 \mathrm{mg} / \mathrm{m}^{2}$ concurrently with radiotherapy were not included. Patients were identified using the ChemoCare (CIS health systems) prescribing system. Any dose modifications and toxicities documented on ChemoCare were recorded. In the event that the reason for a dose reduction was unclear or no toxicities were recorded, the clinic letters and/or clinical notes were reviewed. In cases where patients experienced more than one toxicity, those toxicities with the highest grade or those deemed most likely to be contributing to a subsequent dose reduction were reported.

\section{Results}

Ninety patients received at least 1 dose of capecitabine for the following indications: adjuvant CRC $\mathrm{n}=34(38 \%)$, palliative CRC $\mathrm{n}=21(23 \%)$, palliative breast cancer $\mathrm{n}=35(39 \%)$. The mean number of cycles of treatment received was 8.7 (range 1-38) in all patients and for each treatment indication as follows: adjuvant CRC 6.6 (range 1-8), palliative CRC 6.7 (range 2-21) and palliative breast cancer 12.1 (range 3-38). Sixty nine percent of patients experienced PPE at some point during their treatment (no PPE $n=28(31 \%)$, grade $1 \mathrm{n}=14(16 \%)$ and $\geq$ grade $2 \mathrm{n}=48$ (53\%).

No patients received all of their chemotherapy at the recommended $2500 \mathrm{mg} / \mathrm{m}^{2}$ total daily dose. Thirty-six patients had treatment initiated from the outset with a dose reduction (protocol amendment due to extensive previous chemotherapy and reduced GFR being the most common reasons). PPE was the documented reason for dose reduction in 28 patients (31\%) (Figure 1). The mean and median cycle number for this dose reduction to occur was cycle 4 (Figure 2). The other toxicities accounting for the remaining dose reductions or indeed ceasing capecitabine altogether were: falling performance status/fatigue $\mathrm{n}=11$ (12\%), diarrhoea $\mathrm{n}=8(9 \%)$, deteriorating renal function $\mathrm{n}=4(4 \%)$, possible cardiac complications $n=2(2 \%)$ and mucositis $n=1(1 \%)$. These toxicities were not mutually exclusive.

In addition, 7 (20\%) of the breast cancer patients were converted from a 3 weekly to a 4 weekly alternate dose regimen (1 week on one week off) to minimise PPE but this was only once well established and clinically benefitting from capecitabine treatment. The mean cycle number to convert to a 4 weekly regimen was 10.3 (range 5-18). This

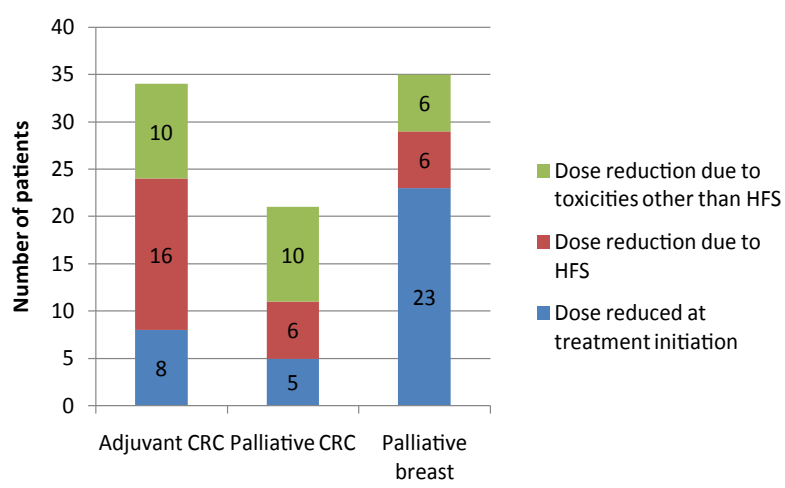

Figure 1: A bar graph to show the reason for the dose reduction (PPE, other toxicities or reduced at treatment initiation*) in patients grouped according to treatment intent.

Note: *indications to commence treatment at a dose reduction include impaired renal function and extensive prior chemotherapy.

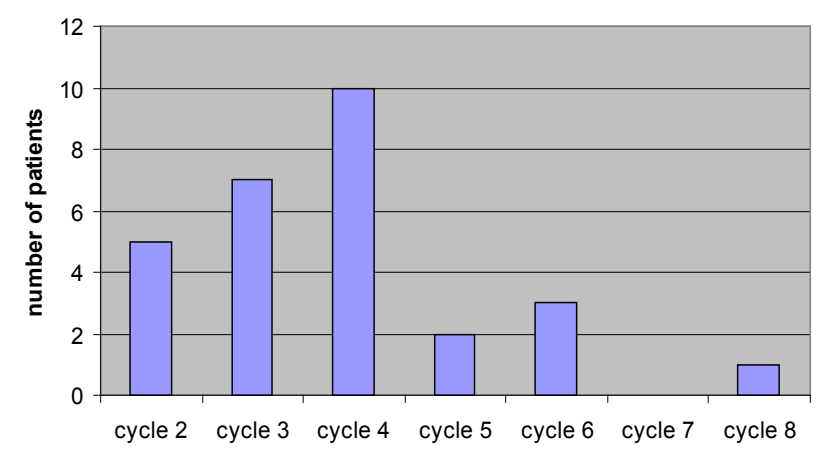

Figure 2: A bar chart to show the cycle number that the dose reduction due to PPE was most likely to occur. 
did allow patients to continue on treatment for a prolonged period of time. The mean number of cycles received in those on a 4 weekly regimen was 19.6 (range 8-38) with 3 patients still continuing on treatment to date.

In the CRC patients, it is noteworthy that PPE contributed towards a dose reduction in a greater proportion of those undergoing treatment with curative rather than palliative intent (47\% versus $29 \%$ ). PPE was the documented sole reason for stopping treatment altogether in 2 of the adjuvant CRC patients, 1 after just 3 cycles and 1 after 7 cycles. It was also deemed to be a contributing factor alongside other toxicities for stopping treatment prematurely in a further 10 of these adjuvant patients.

\section{Discussion}

PPE is a common clinical problem and was the documented reason for a reduction in dose intensity in over one third of patients. This is in keeping with the higher of the levels reported in clinical trials [5].

In the CRC cancer patients, PPE was more likely to lead to a dose reduction in those receiving treatment with curative rather than palliative intent. This was not due to differences in dose of capecitabine at the initiation of treatment as illustrated by the fact that when assessing only those patients commenced on $100 \%$ dose, the incidence of reductions due to PPE in the adjuvant and palliative groups were $61.5 \%$ and $37 \%$ respectively. The mean number of cycles received in the adjuvant and palliative setting was also similar (6.6 versus 6.7) suggesting that finding is also not due to a difference in the duration of treatment. One could speculate that that if the presence of elevated TP expression in the palms of the hands is a causative mechanism for capecitabine-related PPE, [11] then higher activation of the capecitabine within the tumour would lead to lower levels being available for activation within the skin. This would thus render PPE less likely in those with a high disease burden. More work to elicit the exact mechanism behind PPE is needed.

Although this work is a retrospective audit and thus is limited by the quality of documentation in the patient notes, it does provide a real life insight into the significant impact of PPE in the clinic which is as yet poorly reported. We have also not ascertained the incidence and impact of PPE in patients receiving capecitabine as part of a combination regimen. An effective treatment for PPE has the potential to improve not only quality of life but also dose intensity and treatment outcomes for patients receiving capecitabine chemotherapy.

\section{Conclusion}

PPE is common with $69 \%$ of patients on capecitabine monotherapy experiencing symptoms at some point during their treatment. PPE was the documented reason for a reduction in dose intensity in over one third of patients. An effective treatment for PPE has the potential to improve quality of life and outcomes in patients being treated with capecitabine chemotherapy.

\section{References}

1. Miwa M, Ura M, Nishida M, Sawada N, Ishikawa T, et al. (1998) Design of a novel oral fluoropyrimidine carbamate, capecitabine, which generates 5 -fluorouracil selectively in tumours by enzymes concentrated in human liver and cancer tissue. Eur J Cancer 34: 1274-1281.

2. Twelves C, Wong A, Nowacki MP, Abt M, Burris H, et al. (2005) Capecitabine as adjuvant treatment for stage iii colon cancer. N Engl J Med 352: 2696-2704.

3. De Paoli A, Chiara S, Luppi G, Friso ML, Beretta GD, et al. (2006) Capecitabine in combination with preoperative radiation therapy in locally advanced, resectable, rectal cancer: A multicentric phase ii study. Ann Oncol 17: 246-251.
4. Xeloda prescribing information.

5. Cassidy J, Twelves C, Van Cutsem E, Hoff P, Bajetta E, et al. (2002) First-line oral capecitabine therapy in metastatic colorectal cancer: a favorable safety profile compared with intravenous 5-fluorouracil/leucovorin. Ann Oncol 13: 566-575.

6. Baack BR, Burgdorf WH (1991) Chemotherapy-induced acral erythema. J Am Acad Dermatol 24: 457-461.

7. Chu D, Lacouture ME, Fillos T, Wu S (2008) Risk of hand-foot skin reaction with sorafenib: A systematic review and meta-analysis. Acta Oncol 47: 176-186.

8. Jacobi U, Waibler E, Schulze P, Sehouli J, Oskay-Ozcelik G, et al. (2005) Release of doxorubicin in sweat: First step to induce the palmar-plantar erythrodysesthesia syndrome? Ann Oncol 16: 1210-1201.

9. Lai SE, Kuzel T, Lacouture ME (2007) Hand-foot and stump syndrome to sorafenib. J Clin Oncol 25: 341-343.

10. Diasio RB (1998) The role of dihydropyrimidine dehydrogenase (DPD) modulation in 5-fu pharmacology. Oncology 12: 23-27.

11. Milano G, Etienne-Grimaldi MC, Mari M, Lassalle S, Formento JL, et al. (2008) Candidate mechanisms for capecitabine-related hand-foot syndrome. $\mathrm{Br} \mathrm{J}$ Clin Pharmacol 66: 88-95.

12. Kang YK, Lee SS, Yoon DH, Lee SY, Chun YJ, et al. (2010) Pyridoxine is not effective to prevent hand-foot syndrome associated with capecitabine therapy: Results of a randomized, double-blind, placebo-controlled study. J Clin Oncol 28: 3824-3829.

13. National Institute of Health (2010).

14. Zhou Y, Peng L, Li Y, Chen L (2013) Prophylactic pyridoxine was not able to reduce the incidence of capecitabine-induced hand-foot syndrome: A metaanalysis. Biomed Rep 1: 873-878.

15. Wolf SL, Qin R, Menon SP, Rowland KMJ, Thomas S, et al. (2010) Placebocontrolled trial to determine the effectiveness of a urea/lactic acid-based topical keratolytic agent for prevention of capecitabine-induced hand-foot syndrome: North central cancer treatment group study N05C5. J Clin Oncol 28: 51825187.

16. Hofheinz RD, Gencer D, Schulz H, Stahl M, Hegewisch-Becker S, et al. (2015) Mapisal versus urea cream as prophylaxis for capecitabine-associated handfoot syndrome: A randomized phase iii trial of the aio quality of life working group. J Clin Oncol 33: 2444-2449.

17. Zhang RX, Wu XJ, Lu SX, Pan ZZ, Wan DS, et al. (2011) The effect of cox-2 inhibitor on capecitabine-induced hand-foot syndrome in patients with stage ii/ iii colorectal cancer: A phase ii randomized prospective study. J Cancer Res Clin Oncol 137: 953-957.

18. Zhang RX, Wu XJ, Wan DS, Lu ZH, Kong LH, et al. (2012) Celecoxib can prevent capecitabine-related hand-foot syndrome in stage ii and iii colorectal cancer patients: Result of a single-centre, prospective randomized phase iii trial. Ann Oncol 23: 1348-1353.

19. O'shaughnessy J, Miles D, Vukelja S, Moiseyenko V, Ayoub JP, et al. (2002) Superior survival with capecitabine plus docetaxel combination therapy in anthracycline-pretreated patients with advanced breast cancer: Phase iii trial results. J Clin Oncol 20: 2812-2823.

20. Traina TA, Theodoulou M, Feigin K, Patil S, Tan KL, et al. (2008) Phase i study of a novel capecitabine schedule based on the Norton-Simon mathematical model in patients with metastatic breast cancer. J Clin Oncol 26: 1797-1802.

21. Zielinski C, Gralow J, Martin M (2010) Optimising the dose of capecitabine in metastatic breast cancer: Confused, clarified or confirmed? Ann Oncol 21: 2145-2152. 